\title{
Kinetics and Morphological Instabilities of Stressed Solid-Solid Phase Transformations
}

\author{
N. G. Rudawski* and K. S. Jones \\ Department of Materials Science and Engineering, University of Florida, Gainesville, Florida 32611-6400, USA \\ R. Gwilliam \\ Nodus Accelerator Laboratory, Advanced Technology Institute, Surrey Ion Beam Centre, Guildford, Surrey GU2 7XH, \\ United Kingdom
}

(Received 29 October 2007; published 21 April 2008)

\begin{abstract}
An atomistic model of the growth kinetics of stressed solid-solid phase transformations is presented. Solid phase epitaxial growth of (001) Si was used for comparison of new and prior models with experiments. The results indicate that the migration of crystal island ledges in the growth interface may involve coordinated atomic motion. The model accounts for morphological instabilities during stressed solid-solid phase transformations.
\end{abstract}

DOI: 10.1103/PhysRevLett.100.165501

PACS numbers: 61.72.uf, 61.43.Dq, 68.55.A-

The study of stressed solid-solid phase transformations has been a topic of fundamental and technological importance for several years [1-3]. However, there are inconsistencies between understanding the atomistic nature of solid-solid phase transformations and the current model of stress-dependent growth. Current atomistic theory suggests growth occurs via nucleation of two-dimensional crystal islands of the growing phase with subsequent migration of island ledges in the interface between the two phases [4,5]. However, the current stress-dependent growth model does not address this and assumes growth occurs via a single, unspecified atomistic process [6]. In this Letter, a model of stress-dependent growth is presented to account for new experimental observations of stress-dependent growth kinetics in conjunction with the current understanding of the atomistic nature of growth.

The current model used to describe the influence of a stress state, $\sigma_{i j}$, on the macroscopic velocity, $v$, of an advancing growth interface is given by the activation strain tensor, $\Delta V_{i j}^{*}=k T \partial \ln (v) / \partial \sigma_{i j}$, given by

$$
v=v(0) \exp \left(\frac{\Delta V_{i j}^{*} \sigma_{i j}}{k T}\right),
$$

where $v(0)$ is the stress-free velocity, $k T$ has the usual meaning, and $i$ and $j$ refer to axes in the coordinate frame of reference [6]. By convention, 1 and 2 are the in-plane directions, 3 is the growth direction, and positive (negative) elements of $\sigma_{i j}$ are tensile (compressive). The basis for Eq. (1) is the observation that for many solid-solid phase transformations, $v(0)=v_{0} \exp \left(-\Delta G^{*} / k T\right)$ over a wide temperature range where $v_{0}$ is a temperature-independent prefactor and $\Delta G^{*}$ is the activation energy for macroscopic growth as given by transition state theory (TST) [7,8]. However, Eq. (1) assumes a single, unspecified atomistic process is responsible for growth thus contradicting current understanding of solid-solid phase transformations [4,5]. Hence, it is assumed $\Delta V_{i j}^{*}=\Delta V_{i j}^{n}+\Delta V_{i j}^{m}$, where $\Delta V_{i j}^{n}$ and $\Delta V_{i j}^{m}$ are the activation strain tensors associated with nucleation and migration processes [6]. The use of $\Delta V_{i j}^{*}$ is accepted for stressed solid phase epitaxial growth (SPEG) of (001) Si amorphized via ion-implantation $[1,6,9,10]$. However, using new results of stressed SPEG of (001) Si as a model system, a model of stressed solid-solid phase transformations is advanced which isolates the nucleation and migration processes. The results suggest that coordinated atomic motion may play a role in island ledge migration.

In this study, a polished $50 \mu$ m-thick (001) $\mathrm{Si}$ wafer was $\mathrm{Si}^{+}$-implanted at 50,100, and $200 \mathrm{keV}$ to doses of $1 \times$ $10^{15}, 1 \times 10^{15}$, and $3 \times 10^{15} \mathrm{~cm}^{-2}$. Subsequently, samples were cleaved along $\langle 110\rangle$ directions into $\sim 0.2 \times 1.8 \mathrm{~cm}^{2}$ strips (with 1 and 2 directions taken to be [110] and [1 10$]$ crystal directions) and uniaxially-stressed up to magnitude of $1.3 \pm 0.1 \mathrm{GPa}$ along [110] $\left(\sigma_{11}\right)$ as presented elsewhere [11]. Stress-free, tensilely stressed, and compressively stressed strips were annealed simultaneously at $525^{\circ} \mathrm{C}$ in $\mathrm{N}_{2}$ ambient for 1.0-4.0 h. No detectable stress relaxation occurred during annealing. Growth was examined using weak-beam dark-field (WBDF) cross-sectional transmission electron microscopy (XTEM). XTEM specimens $\sim 10 \mu \mathrm{m}$ long were prepared via focused ion beam milling within a lateral distance of $\pm 3 \mathrm{~mm}$ from the strip centers to minimize the presence of any thermal gradient. Because of the very small specimen length to strip length ratio, it is reasonably assumed no intraspecimen stress gradients existed.

Figure 1(a) presents a WBDF-XTEM image of the asimplanted structure indicating an initial amorphous $(\alpha) \mathrm{Si}$ layer $350 \pm 5 \mathrm{~nm}$ thick continuous to the surface as confirmed using selected area diffraction. Following annealing for $4.0 \mathrm{~h}$ with $\sigma_{11}=0$, shown in Fig. 1(e), $138 \pm 5 \mathrm{~nm}$ of growth occurred with the error in all growth measurements given as the root-mean squared roughness of the $\alpha /$ crystalline (growth) interface. End of range (EOR) de- 


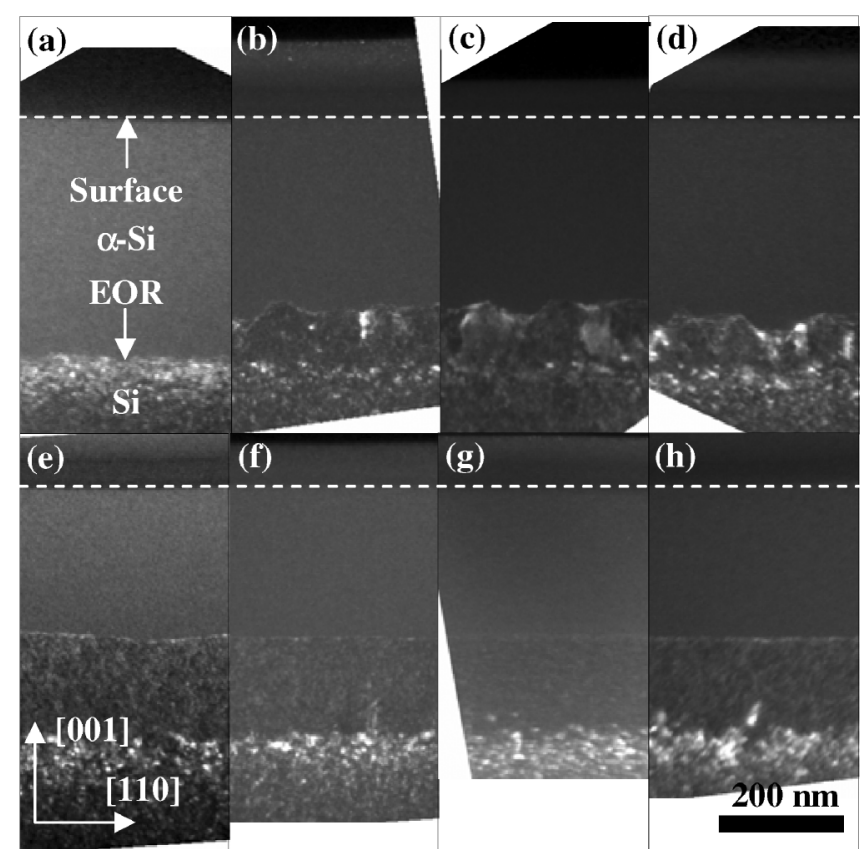

FIG. 1. (a) WBDF-XTEM image of the as-implanted structure. WBDF-XTEM images of specimens after annealing for $4.0 \mathrm{~h}$ with $\sigma_{11}=$ (b) -0.5 , (c) -1.0 , (d) -1.3 , (e) 0 , (f) 0.5, (g) 1.0, and (h) $1.3 \mathrm{GPa}$.

fects were observed near the initial $\alpha$ /crystalline interface for all specimens [12]. As reported by others, these defects do not interact with the growth interface and do not influence SPEG kinetics $[8,12]$. In the cases of $\sigma_{11}=-0.5$, -1.0 , and $-1.3 \mathrm{GPa}$, shown in Figs. 1(b) $-1(\mathrm{~d}), 77 \pm 9$, $72 \pm 8$, and $68 \pm 9 \mathrm{~nm}$ of growth occurred which is less than the $\sigma_{11}=0$ case. Growth interface roughening was observed with $\sigma_{11}<0$, consistent with prior reports [911]. For cases of $\sigma_{11}=0.5,1.0$, and $1.3 \mathrm{GPa}$, shown in Figs. 1(f) $-1(\mathrm{~h}), 138 \pm 6 \mathrm{~nm}$ of growth occurred, similarly to the $\sigma_{11}=0$ case. The surface-EOR distance showed no detectable variation between as-implanted and annealed specimens indicating no detectable flow of $\alpha$-Si [13]. Annealing of specimens for 1.0, 2.0, and $3.0 \mathrm{~h}$ was performed for all values of $\sigma_{11}$ and the resulting amounts of growth measured (not presented). From this data, $v$ versus $\sigma_{11}$ was calculated as presented in Fig. 2. No detectable difference was observed in $v$ for $0 \leq \sigma_{11} \leq 1.3 \mathrm{GPa}$ with $v=34 \pm 2 \mathrm{~nm} / \mathrm{h}$. In compression, $v$ was retarded to a limiting rate of $17 \pm 2 \mathrm{~nm} / \mathrm{h}$ for $-1.3 \leq \sigma_{11} \leq-0.5$, while $\sigma_{11}=-0.25 \mathrm{GPa}$ retarded $v$ to $22 \pm 2 \mathrm{~nm} / \mathrm{h}$. The predicted [6] behavior of $v$ versus $\sigma_{11}$ is provided for reference. No predicted increases in $v$ were observed for $0<\sigma_{11}$ while retardation for $\sigma_{11}<0$ was consistent with prior work [6,9-11]. However, in compression, the retardation appears to reach a limit and the reductions are greater than predicted.

Growth results from nucleation of two-dimensional crystal islands and subsequent in-plane migration of island ledges $[4,5]$. The rate of island nucleation, $\tau_{n}^{-1}$, is a scalar

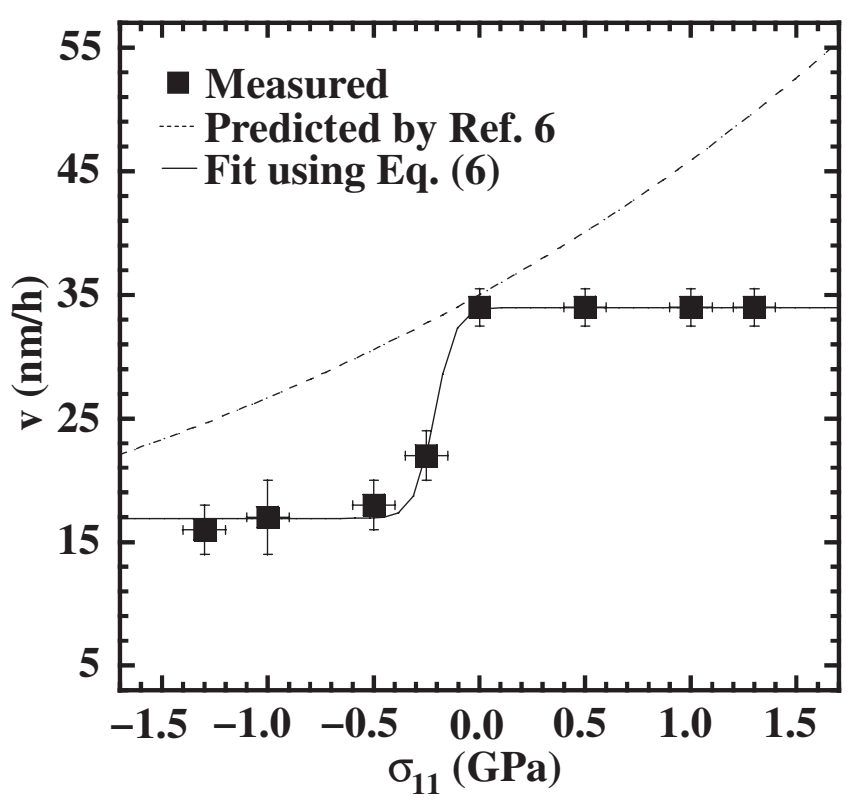

FIG. 2. Plot of $v$ versus $\sigma_{11}$.

property while the rate of ledge migration, $\tau_{m, i j}^{-1}$, is a second order tensor property analogous to diffusivity given by

$$
\tau_{m, i j}^{-1}=\left(\begin{array}{cc}
\tau_{m, 11}^{-1} & 0 \\
0 & \tau_{m, 11}^{-1}
\end{array}\right),
$$

for the $\alpha$-Si/(001) Si interface for $\sigma_{i j}=0$. The velocity of ledge migration is given by $v_{m, i}=\tau_{m, i j}^{-1} \Delta x_{j}$, where $\Delta x_{j}$, is the ledge migration vector dependent on the crystallography of the interface and the coordinate frame of reference (in this case, $\Delta x_{1}=\Delta x_{2}=0.38 \mathrm{~nm}$ ) with both $v_{m, 1}$ and $v_{m, 2}$ contributing to $v$. Furthermore, $v_{m, i}$ is not subject to any symmetry requirements and thus $\boldsymbol{v}_{m, 1}$ and $\boldsymbol{v}_{m, 2}$ are reasonably assumed independent of one another. The nucleation of crystal islands with monolayer thickness $\Delta x$ $(0.14 \mathrm{~nm})$ causes additional contribution to $v$. However, nucleation and migration are sequential and both must be accomplished for growth to proceed. Thus, $v$ is given by

$$
v=\frac{1}{\frac{1}{\tau_{n}^{-1} \Delta x}+\frac{1}{\tau_{m, 1}^{-1} \Delta x_{1}}}+\frac{1}{\frac{1}{\tau_{n}^{-1} \Delta x}+\frac{1}{\tau_{m, 11}^{-1} \Delta x_{2}}} .
$$

Both $\tau_{n}^{-1}$ and $\tau_{m, i j}^{-1}$ are presumably Arrhenius processes and can be modified via application of $\sigma_{i j}$ as given by TST [7]. However, since $\tau_{m, i j}^{-1}$ is a tensor property, $\Delta V_{k l}^{m, i j}=$ $k T \partial \ln \left(\tau_{m, i j}^{-1}\right) / \partial \sigma_{k l}$, a fourth-order tensor, describes the change in $\tau_{m, i j}^{-1}$ with respect to $\sigma_{k l}$ where $k$ and $l$ refer to axes in the coordinate frame of reference. Hence,

$$
\tau_{m, i j}^{-1}=\tau_{m, i j}(0)^{-1} \exp \left(\frac{\Delta V_{k l}^{m, i j} \sigma_{k l}}{k T}\right),
$$

where $\tau_{m, i j}(0)^{-1}$ is the $\sigma_{i j}=0$ value of $\tau_{m, i j}^{-1}$. Similar models describe point defect motion in bulk Si [14]. 
Since $\tau_{m, 12}(0)^{-1}=\tau_{m, 21}(0)^{-1}=0$ and the only term in $\sigma_{i j}$ is $\sigma_{11}, \Delta V_{11}^{m, 11}$ and $\Delta V_{11}^{m, 22}$ are the only relevant terms in $\Delta V_{k l}^{m, i j}$. During ledge motion, the greatest in-plane volume change presumably occurs parallel to rather than orthogonal to a given migration direction implying $\left|\Delta V_{11}^{m, 22}\right| \ll\left|\Delta V_{11}^{m, 11}\right|$. Thus, only migration along 1 is significantly altered by $\sigma_{11}$. Regarding $\tau_{n}^{-1}, \Delta V_{i j}^{n}=$ $k T \partial \ln \left(\tau_{n}^{-1}\right) / \partial \sigma_{i j}$ describes the change in $\tau_{n}^{-1}$ with respect to $\sigma_{i j}$ given by

$$
\Delta V_{i j}^{n}=\left(\begin{array}{ccc}
\Delta V_{11}^{n} & 0 & 0 \\
0 & \Delta V_{11}^{n} & 0 \\
0 & 0 & \Delta V_{33}^{n}
\end{array}\right),
$$

for the $\alpha-\mathrm{Si} /(001) \mathrm{Si}$ interface. The nucleation of a crystal island causes volume change primarily in the growth direction rather than the in-plane directions, similarly to the formation of a Si self-interstitial near a surface [14]. Thus, $\Delta V_{11}^{n} \sim 0$ and $\tau_{n}^{-1} \sim \tau_{n}(0)^{-1}$, where $\tau_{n}(0)^{-1}$ is the $\sigma_{i j}=$ 0 nucleation rate. Making the necessary substitutions, $v$ with $\sigma_{11}$ is given by

$$
\begin{aligned}
v= & \frac{\Delta x}{\tau_{n}(0)+2^{-3 / 2} \tau_{m, 11}(0) \exp \left(\frac{-\Delta V_{11}^{m, 11} \sigma_{11}}{k T}\right)} \\
& +\frac{\Delta x}{\tau_{n}(0)+2^{-3 / 2} \tau_{m, 11}(0)} .
\end{aligned}
$$

Equation (6) was fit to the data presented in Fig. 2 and $\tau_{n}(0), \tau_{m, 11}(0)$, and $\Delta V_{11}^{m, 11}$ were calculated to be $29 \pm 1 \mathrm{~s}$, $1.01 \pm 0.01 \mathrm{~s}$, and $(12 \pm 1) \Omega$, where $\Omega$ is the atomic volume of Si. The value of $\tau_{n}(0)$ is nearly 30 times $\tau_{m, 11}(0)$ which is consistent with prior observations suggesting nucleation is slower than migration [5]. The positive value of $\Delta V_{11}^{m, 11}$ suggests in-plane expansion associated with ledge migration. Presumably, this expansion occurs to allow easier bond rearrangement (resulting from the $\alpha$-Si to Si phase transformation) at an island ledge. In the case of $0 \ll \sigma_{11}$, Eq. (6) reduces to the tensile saturation velocity, $v_{t} \approx 2 \Delta x / \tau_{n}(0)$, and growth is limited by nucleation. However, in the case of $\sigma_{11} \ll 0$, Eq. (6) reduces to the compressive limit velocity, $v_{c} \approx \Delta x / \tau_{n}(0)$, and growth is still nucleation limited. Thus, it is predicted that $v_{t} / v_{c} \sim 2$ independently of growth temperature. It is important to note the interfacial roughening with $\sigma_{11}<0$ which generates off-axis growth interfaces which grow up to $\sim 25$ times slower than [001] SPEG [15]. However, this cannot primarily account for the retardation with $\sigma_{11}<0$ since $v_{t} / v_{c} \sim 2$.

The $\alpha-\mathrm{Si} /(001) \quad \mathrm{Si}$ interface model advanced by Spaepen [4] is used as a basis for an atomistic picture of stressed-SPEG in conjunction with the work of Williams et al. [5] suggesting growth is a two-step process. Figure 3 presents atomistic schematics of the SPEG migration process with different $\sigma_{11}$. For $\sigma_{11}=0$, shown in Fig. 3(a), a crystal island nucleates and the crystal ledges migrate (indicated by arrows) rapidly and evenly in both in-plane directions since $\tau_{m, i j}(0)^{-1}$ is isotropic. In the case of $0<$ $\sigma_{11}$, shown in Fig. 3(b), a crystal island nucleates and ledge migration along 1 is enhanced relative to 2 . Thus, nucleation is the limiting step for $0 \leq \sigma_{11}$ since migration is rapid in both directions explaining why $v$ does not change for $0 \leq \sigma_{11}$ contradictory to the predicted [6] behavior. With $\sigma_{11}<0$, shown in Fig. 3(c), migration along 1 is retarded but unchanged along 2 . Thus, the growth rate is effectively halved since only migration along 2 effectively contributes to the growth rate. This explains why $v$ cannot be retarded indefinitely by $\sigma_{11}<0$ as predicted [6].

The presented stressed-SPEG model explains interfacial roughening during stressed SPEG in other material systems [16] as well as interface roughening observed in this and prior studies of stressed Si SPEG [9-11]. A growth interface with perturbations under in-plane macroscopic compression, shown in Fig. 4(a), has localized concentrated in-plane compressive stress $\left(\sigma_{11}^{\text {local }}\right)$ in the troughs of the interface and localized tensile stress in the peaks of the interface, since $\alpha$-Si is near fully relaxed at $525^{\circ} \mathrm{C}$ [13]. Thus, as per Eq. (6), the peaks grow faster than the troughs and the interface roughens. However, the application of macroscopic tension, shown in Fig. 4(b), causes a reversal of the localized stress states which dampens any perturbations in the growth interface due to kinetic limitations. Hence, this model explains observed morphological instabilities during stressed solid-solid phase transformations.

Interestingly, the actual data from prior work [6] does show that $v$ increases (retards) with $0<\sigma_{11}\left(\sigma_{11}<0\right)$ in some (though not all) specimens. However, though Eq. (1) seems to describe the data from Ref. [6], there are many significant deviations in the data from this trend and the

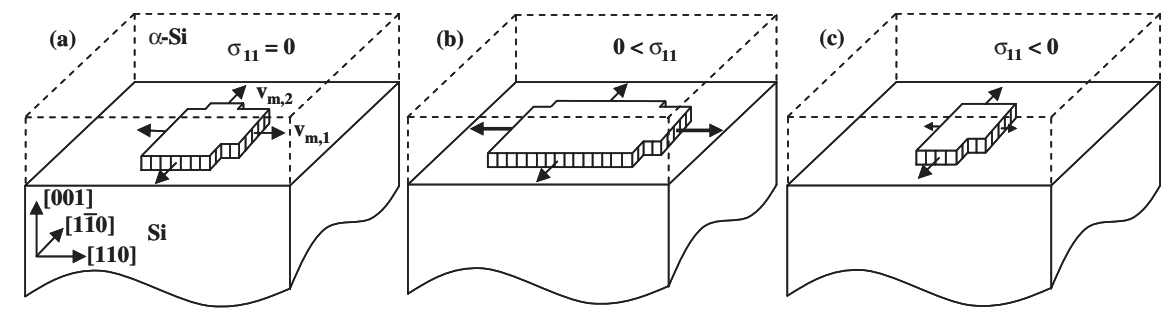

FIG. 3. Atomistic schematics of the in-plane SPEG migration process with (a) $\sigma_{11}=0$, (b) $0<\sigma_{11}$, and (c) $\sigma_{11}<0$. 


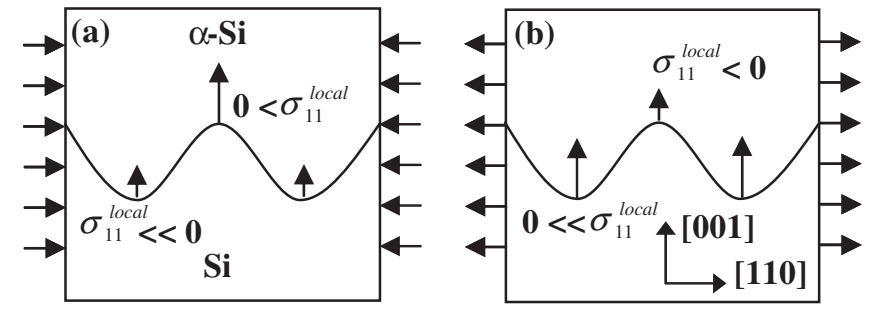

FIG. 4. Schematic representations of growth interface morphological stability with in-plane macroscopic (a) compression and (b) tension.

results as a whole are somewhat weakly ordered. It is mentioned in Ref. [6] that intrasample thermal fluctuations may have influenced the results and the analysis techniques used were less site-specific relative those used in this study giving rise to the possible influence of intraspecimen stress gradients. Also, $\left|\sigma_{11}\right| \leq 0.6 \mathrm{GPa}$ was applied in prior work [6] which is smaller than the range used in this study. However, while these issues may explain why the scatter in the presented work is less than prior work, it is important to note Aziz et al. [6] were the first to observe and model stress-influenced SPEG kinetics.

Furthermore, the prior model [6] of growth occurring via a single, unspecified process is a reasonable theory to explain the somewhat scattered results observed in Ref. [6]. However, Eq. (6) provides an equally reasonable explanation for the prior data and the model of Ref. [6] cannot reasonably explain the results presented in this study. Thus, consideration of the nucleation and migration processes during growth is necessary to explain the new results.

Importantly, the results provide suggestions as to the atomistic nature of migration during solid-solid phase transformations. In particular, the large value of $\Delta V_{11}^{m, 11}$ suggests that in-plane ledge motion is coordinated and involves the lateral advancement of multiple atoms along a growing island ledge rather than individual atomic motion as originally suggested [6]. Analogous coordination is observed in the deformation of metals which is typically characterized by activation volumes on the order of $\sim 100$ times the atomic volume of the material $[17,18]$. Thus, $\Delta V_{11}^{m, 11}=(12 \pm 1) \Omega$ is reasonable considering migration may be coordinated and not due to independent atomic motion.

In summary, this study of stressed-SPEG of (001) Si strongly suggests the currently accepted model of stressed solid-solid phase transformations cannot reasonably explain the stress-dependent growth kinetics observed in this study. To explain these results, an atomistic model of stressed solid-solid phase transformation kinetics is advanced which considers growth to be mediated by crystal island nucleation with subsequent island ledge migration with applied stress altering each process differently. This approach of isolating nucleation and migration processes not only explains the observed growth kinetics, but also morphological instabilities observed in this and prior studies of stressed solid-solid phase transformations. The results also suggest that in-plane migration may involve coordinated motion of atoms along a growing island ledge.

The authors acknowledge the Semiconductor Research Corporation for funding this research and the Major Analytical Instrumentation Center at the University of Florida for use of the transmission electron microscope and focused ion beam facilities.

*Corresponding author. ngr@ufl.edu

[1] E. Nygren, M. J. Aziz, and D. Turnbull, Appl. Phys. Lett. 47, 232 (1985).

[2] G. Q. Lu et al., Appl. Phys. Lett. 56, 137 (1990).

[3] Q.Z. Hong et al., J. Appl. Phys. 71, 1768 (1992).

[4] F. Spaepen, Acta Metall. 26, 1167 (1978).

[5] J.S. Williams et al., Phys. Rev. Lett. 55, 1482 (1985).

[6] M. J. Aziz, P. C. Sabin, and G.-Q. Lu, Phys. Rev. B 44, 9812 (1991).

[7] S. Glasstone, K. J. Laidler, and H. Eyring, The Theory of Rate Processes (McGraw-Hill, New York, 1948).

[8] G. L. Olsen and J. A. Roth, Mater. Sci. Rep. 3, 1 (1988).

[9] W. Barvosa-Carter et al., Phys. Rev. Lett. 81, 1445 (1998).

[10] W. Barvosa-Carter and M. J. Aziz, Appl. Phys. Lett. 79, 356 (2001).

[11] N. G. Rudawski, K. S. Jones, and R. Gwilliam, Appl. Phys. Lett. 91, 172103 (2007).

[12] K. S. Jones, S. Prussin, and E. R. Webber, Appl. Phys. A 45, 1 (1988).

[13] C. A. Volkert, J. Appl. Phys. 70, 3521 (1991).

[14] M. J. Aziz, Appl. Phys. Lett. 70, 2810 (1997).

[15] L. Csepregi, E. F. Kennedy, and J. W. Mayer, J. Appl. Phys. 49, 3906 (1978).

[16] R. G. Elliman and W. C. Wong, Appl. Phys. Lett. 69, 2677 (1996).

[17] R. J. Asaro and S. Suresh, Acta Mater. 53, 3369 (2005).

[18] Y. M. Wang, A. V. Hamza, and E. Ma, Acta Mater. 54, 2715 (2006). 